\title{
Contribution of the calf pump and foot pump to the return of venous blood from the lower extremity
}

\section{Recek ${ }^{1}$}

${ }^{1}$ Retired, formerly Department of Surgery, University Hospital, Hradec Kralove, Czech Republic. Address: Cestmir Recek, Mantlergasse 24, 1130 Vienna, Austria.

submitted: Jan 4, 2018, accepted: Jan 11, 2018, EPub Ahead of Print: Jan 14, 2018, published: Jun 30, 2018

Conflict of interest: None

DOI: 10.24019/jtavr.43 - Corresponding author: Dr. Cestmir Recek, cestmir@recek.at

(C) 2017 Fondazione Vasculab impresa sociale ONLUS. All rights reserved.

\begin{abstract}
The anatomy and function of the calf pump and the foot pump as well as the interplay of their activity are described. The calf muscle pump constitutes an effective mechanism enhancing efficiently the return of venous blood toward the heart. During calf muscle contractions, the venous blood is ejected mainly into the popliteal vein, but a smaller part escapes through calf perforators into the saphenous system and streams further in the centripetal, i.e. physiological direction toward the heart. Calf muscle contractions induce marked increase in systolic pressure and calf muscle relaxations entail decrease of diastolic pressure both in deep and superficial veins of the lower leg. The systolic and diastolic pressure changes are produced in deep veins and are transmitted through calf perforators into the saphenous system, as documented by simultaneous pressure recordings in the posterior tibial and great saphenous veins. The systolic increase of pressure in
\end{abstract}

\section{Introduction}

The vis a tergo, the remainder of the cardiac energy propelling venous blood from the lower extremities against the gravitation toward the heart is not powerful enough to move the venous blood toward the heart effectively; the pressure difference between the venules and the right atrium amounts to about $20 \mathrm{~mm} \mathrm{Hg}$. In addition to this motive force, the calf muscle pump (CMP) constitutes an effective mechanism that promotes drainage of venous blood from the lower extremity, produces marked increase of systolic pressure and decrease of diastolic pressure both in deep and superficial veins of the lower leg, and entails a distinct physiological decrease of ambulatory venous pressure in the lower leg veins. The requirements for a proper action the great saphenous vein is caused by the outward flow within calf perforators; competent valves in calf perforators would preclude any relevant pressure increase. Calf pump activity entails a distinct decrease of ambulatory venous pressure in lower leg veins, whereas in the thigh veins the pressure does not decrease; in this way, the ambulatory pressure gradient of $37.4+-6.4 \mathrm{~mm} \mathrm{Hg}$ arises between thigh and lower leg veins and triggers the venous reflux in incompetent venous channels connecting both poles of the ambulatory pressure gradient. In contrast to the very efficient performance of the calf pump, the performance of the foot pump is hemodynamically unimportant. The ejection volume produced by the calf muscle pump comes at about $60 \mathrm{ml}$ or more, whereas the blood volume ejected by the foot pump reaches a negligible value of 3-4 $\mathrm{ml}$.

Keywords calf muscle pump; foot pump

of the CMP are patency and perfect competence of deep lower leg veins, no impediments in the venous outflow tract, and undisturbed contractility of the calf musculature. The physiological effectivity of the CMP is compromised by the venous reflux since the volume of the venous blood expelled by the CMP is more or less replaced by the refluxing volume. The contribution of the foot pump to the venous return from the lower extremity has not so far been cleared up.

The intention of this review article is to evaluate the physiological significance of the CMP and the foot pump, to assess the systolic and diastolic pressure behavior in deep and superficial veins of the lower leg during calf pump activity, as well as to evaluate the flow behavior in calf 
perforators resulting from systolic and diastolic pressure differences.

\section{Anatomy and function of the calf muscle pump}

Calf muscle pump is formed by the calf musculature and the deep lower leg veins. The calf musculature consists of

- $\quad$ 1. The posterior compartment inducing plantar flexion of the ankle joint and the toes as well as supination of the foot,

2. The anterior compartment inducing dorsal flexion of the ankle joint and the toes, and

3. The lateral compartment inducing plantar flexion of the ankle joint as well as pronation of the foot.

Deep lower leg veins consist of three paired conductive veins: the posterior tibial vein (PTV), anterior tibial vein, and peroneal vein, as well as of the muscular veins contained in the sural musculature (most important: gastrocnemius veins and soleus veins). Calf muscle pump is called "peripheral heart": contractions of the sural muscles eject the venous blood centripetally toward the heart and promote substantially the return of venous blood from the lower extremity against the gravitation force. During calf muscle contraction, the pressure rises considerably in deep lower leg veins (on average by $75 \mathrm{~mm} \mathrm{Hg}$ ) and a little less also in the great saphenous vein (GSV). The systolic pressure difference between the PTV and the GSV amounts in healthy people to about $40 \mathrm{~mm} \mathrm{Hg}$ and in varicose vein patients to merely $14 \mathrm{~mm} \mathrm{Hg}^{1-3}$; the different pressure gradient is apparently caused by the unequal flow rate capacity of the calf perforators in healthy people and in varicose vein patients. Thus, during calf muscle contractions the venous blood is ejected mainly into the popliteal vein, but a smaller part escapes through calf perforators into the GSV and streams further in the centripetal direction toward the heart. During calf muscle relaxation, the diastolic pressure decreases both in deep lower leg veins and in the $\mathrm{GSV}^{1,2,4}$; the pressure gradient turns round, the diastolic pressure in the GSV is roughly $13 \mathrm{~mm} \mathrm{Hg}$ higher than in the $\mathrm{PTV}^{2,3}$. The systolic and diastolic pressure changes are produced in deep veins. Pressure transmissions between the PTV and the GSV take place through the connecting channels, through calf perforators, which was documented by simultaneous pressure recordings. The pressures transmissions could not take place without bidirectional streaming through calf perforators, i.e. without systolic outward and diastolic inward flow. The steep systolic increase in pressure in the GSV is produced by the outward flow through calf perforators. This flow is centripetal, i.e. physiological; it continues via GSV toward to heart and can be registered in the GSV by duplex ultrasonography. If calf perforators in healthy people were competent, the increase in systolic pressure in the GSV, moreover coincident with similar steep increase in the PTV could not occur. Similar conditions exist in the heart across the competent and incompetent mitral valve. Competent mitral valve precludes systolic increase in pressure in the left atrium. Regurgitation through incompetent mitral valve induces increase in pressure in the left atrium. Thus, with some benevolence, CMP can be compared with the left ventricle, the saphenous system with the left atrium. The pressure in the popliteal and femoral vein oscillates during the CMP activity but does not decrease below the value of the hydrostatic pressure during the diastolic phase ${ }^{1,2,4}$. The pressure difference arising in this way between the popliteal/femoral vein and deep lower leg veins has been called ambulatory pressure gradient; its value is $37.4+-6.4 \mathrm{~mm} \mathrm{Hg}^{5}$. Ambulatory pressure gradient triggers reflux in incompetent veins connecting both poles of the pressure gradient.

The performance of the CMP depends primarily on the patency and impeccable competence of the deep conductive veins of the lower leg (posterior and anterior tibial veins and peroneal veins). Incompetence of these veins disturbs the performance of the CMP. Further factors impairing CMP performance are obstacles in the outflow venous $\operatorname{tract}^{6}$ and reduced calf muscle strength due to muscle weakness, atrophy or palsy.

Plethysmographic findings enable quantification of the CMP performance. Ejection volume produced by the CMP is an important parameter indicating its effectivity; it is determined by air-plethysmography; the normal values are in the range of $60-90 \mathrm{ml}^{7}$. Ejection fraction is another parameter used for evaluating CMP performance; it is expressed in percentage and is calculated as the volume of blood ejected with one calf muscles contraction divided by the functional venous volume. Because this parameter depends on the value of the functional venous volume, it is not suitable for evaluating results after therapeutic procedures that reduce the venous volume contained in varicose veins. The ejection fraction, i.e. the quotient $\mathrm{EV} / \mathrm{VV}$ improves and simulates improvement of the calf muscle performance but the pumping effectivity remains actually unaffected. Other valuable plethysmographic parameters registered after a series of calf muscle contractions and relaxations, such as refill time t-50 and t-90, refill volume gained by strain gauge plethysmography, and residual volume gained by air plethysmography are influenced both by the CMP performance and by the venous reflux. Saphenous reflux simulates malfunction of the CMP because the ejection volume is devaluated by the refluxing volume. Therefore, the lastly mentioned parameters can be taken into consideration for evaluation of the CMP performance only after elimination of saphenous reflux. As a rule, in patients with primary varicose veins even the severest 
hemodynamic disorders in CEAP classes C4 - C6 are caused by the saphenous reflux, whereas the performance of the CMP remains unaffected. After elimination of saphenous reflux physiological ambulatory venous pressure conditions as well as normal plethysmographic values are restored, which would be impossible if impairment of CMP performance were present ${ }^{3,8-11}$;

\section{Anatomy and function of the foot pump}

The substantial part of venous reservoir of the foot pump is formed by the plantar venous plexus. According to Binns and Pho ${ }^{12}$ the plantar venous plexus consists, in addition to subtle veins, mainly of the large lateral plantar vein that is partly double-barreled, of a little smaller medial plantar vein, and of the deep plantar venous arch. The plantar veins are convoluted and fitted with valves directing the flow into the PTV. From the diameter and length of these veins it can be computed that the plantar venous plexus contains altogether about $6 \mathrm{ml}$ of blood. In another study, White et al. ${ }^{13}$ found on the average 2.7 large veins in the plantar venous plexus with a diameter of $4.0+-1.0 \mathrm{~mm}$. The entire length of these veins along the forefoot amounts to about $30 \mathrm{~cm}$; that means they contain about $5 \mathrm{ml}$ of blood. So, the total venous volume of the sole including the blood volume in the foot muscles might hardly reach $10 \mathrm{ml}$.

The contribution of the foot pump to the venous return is still disputable. Plantar veins empty due to compression exerted by weight bearing. Because of the anatomical configuration of the foot only the lateral part of the foot is involved in the pumping process. If taking into account the normal ejection fraction of $60 \%$, the estimated ejection volume of the foot pump would come at about $3-4 \mathrm{ml}$; the large part of this very small volume is ejected into deep lower leg veins, a petty part might be ejected into the GSV.

Broderick et al. ${ }^{14}$ researched venous emptying from the foot using several provoking maneuvers: weight bearing, toe curl, electrical stimulation of the tibial nerve at the medial malleolus, and passive compression. Flow measurements in the particular lower leg veins (posterior tibial veins, anterior tibial veins, and peroneal veins) as well as in the popliteal veins were performed using DUS; the flow in the GSV was not measured. Flow measurements in the deep lower leg veins were performed at the level between the lower and middle part of the lower leg. Values of the ejection volume were obtained by multiplying the cross-sectional area $\mathrm{X}$ mean velocity $\mathrm{X}$ total flow duration. Electrical stimulation of the foot muscles ejected just $1 \mathrm{ml}$ of venous blood into the lower leg veins altogether, which documents that the contraction of the metatarsal muscles exerts just a miniscule effect. Intermittent pneumatic compression of the foot produced venous outflow of $2.18 \mathrm{ml}$, which is likewise negligible. Weight bearing maneuver expelled $6.08 \mathrm{ml}$ and toe curls
$5.03 \mathrm{ml}$ of venous blood into deep lower leg veins. Oddly enough, weight bearing maneuver and toe curls induced in the popliteal vein a remarkable centripetal flow of 33.34 $\mathrm{ml}$ and $31.04 \mathrm{ml}$, respectively, which evidenced that weight bearing maneuvers and toe curls activated not only the foot pump but more effectively also the calf muscle pump. It is obviously quite difficult to provoke selectively the activity of the foot pump without evoking in the weigh-bearing limb involuntary contractions of the calf musculature that occur in order to ensure balance of the body. In addition, toe curls activate by itself the calf muscle pump (musculus flexor hallucis longus and musculus flexor digitorum longus). Because the flow measurements in the lower leg veins were performed at the level between the lower and middle third of the lower leg, it can be deduced that the upper section of the calf musculature where the substantial mass of calf musculature is concentrated might be the most important part of the CMP.

The findings documented that intermittent pneumatic compression of the sole and active contractions of the foot muscles were not capable of expelling a noteworthy volume of venous blood from the foot. The total volume ejected by these two maneuvers into deep lower leg veins amounted to $3.18 \mathrm{ml}(1 \mathrm{ml}$ due to electrical stimulation of the tibial nerve, $2.18 \mathrm{ml}$ due to intermittent pneumatic compression), which complies with the calculations of the venous capacity and ejection potential derived from the anatomical findings, as mentioned in the foregoing paragraph. In addition, the results of these measurements evidenced that the weight bearing maneuver that was primary intended to activate the foot pump was able to achieve only $20 \%$ of the ejection volume registered in the popliteal vein, whereas $80 \%$ of the ejection volume in the popliteal vein accrued from the upper two parts of the calf pump (proximal to the probes measuring the flow in deep lower leg veins). If we take into account that the foot ejected just $3 \mathrm{ml}$ of venous blood into deep lower leg veins but the weight bearing maneuver induced ejection of $6.08 \mathrm{ml}$ registered at the point of measurements in deep lower leg veins, it follows that the lower part of the calf muscles (below the probes) contributed about $3 \mathrm{ml}$ to the ejection volume. Considering the fact that the ejection volume produced by the CMP activity comes at not less than $60 \mathrm{ml}$, it is clear that 3 $\mathrm{ml}$ ejected by the foot pump are negligible and could be ignored.

\section{Interplay of the calf and foot pump activity}

The calf and foot pumps work probably in sequence during normal walking: calf pump (extensor muscles inducing dorsiflexion in the ankle joint) -> foot pump (foot in contact with the ground) $->$ calf pump (flexor muscles inducing plantar flexion in the ankle joint). When performing tip toe maneuvers, the toe stand represents the systole of the calf muscle pump; the return to the stand on the heel represents the diastole. However, treading fully 
on the heel activates simultaneously the foot pump. Thus, in contrast to the gait cycle, during the tip toe maneuvers the systolic phase of the foot pump occurs during the diastolic phase of the calf pump, i.e. it works de facto as an interference factor. The effect can be demonstrated on the pressure curves. Figure 1 depicts simultaneous pressure recordings in the PTV and GSV in a patient with a severe saphenous reflux; no decrease in pressure occurred during calf pump activity. On the descending arms of the pressure curves, i.e. during the diastole of the calf pump petty spikes were registered, which were apparently evoked by the systolic input of the foot pump. It is evident that the systolic phase of the foot pump does not affect the systolic/diastolic amplitudes produced by the calf pump; the recordings document that the hemodynamic effect of the foot pump is negligible in comparison with the calf pump. This is well comprehensible because the venous blood volume contained in the foot is irrelevant in comparison with the blood volume contained in the calf. In addition, the foot pump is not able to produce the pronounced diastolic decrease in pressure either in the PTV or in the GSV. The systolic and diastolic pressure amplitudes in the PTV and GSV appear synchronously and are very similar, which documents that they are produced by the calf pump, both in varicose vein patients and in healthy people.

\section{Conclusion}

Calf muscle pump is an effective mechanism propelling venous blood from the lower extremities against the gravitation force toward the heart; the contribution of the foot pump is in this regard negligible. The marked and synchronous increase in systolic and decrease in diastolic pressure in the PTV and the GSV both in varicose vein patients and in healthy people is caused by free pressure transmission and bidirectional flow through the calf perforators, which makes deep and superficial veins of the lower leg conjoined vessels.

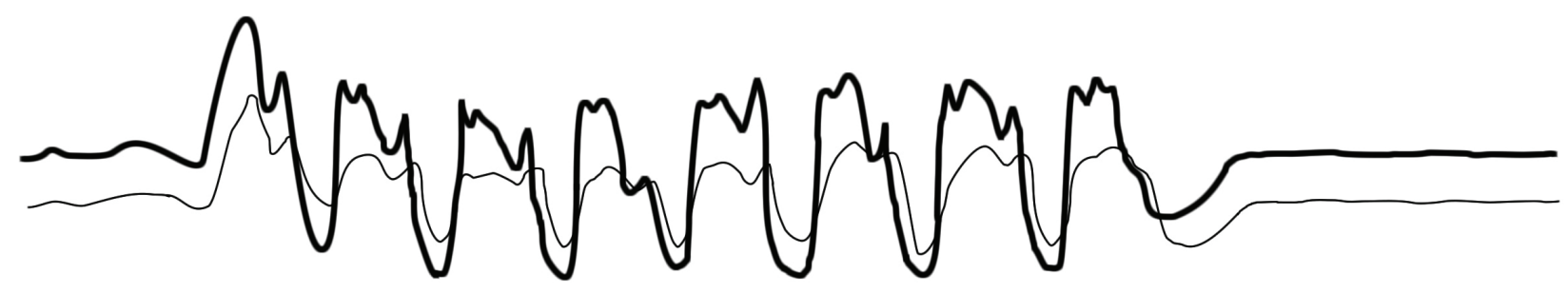

Figure 1 - Simultaneous pressure recordings in the PTV (upper bold curve) and the GSV (lower thin curve) were performed during tiptoe movements in a patient with primary varicose veins and a strong saphenous reflux. The severest hemodynamic disturbance with no decrease in ambulatory venous pressure was registered. Marked systolic and diastolic amplitudes caused by the calf pump are discernible. Little spikes were generated on the descending arms of the pressure curves; they represent the systolic contribution of the foot pump; they do not disturb the decrease in pressure during the calf pump diastole either in the PTV or in the GSV. (Examination was performed by the author himself in the Dept. of Surgery, University Hospital, Hradec Kralove, Czech Rep.).

\section{References}

1) Arnoldi CC. Venous pressure in the legs of healthy human subjects at rest and during muscular exercise in nearly erect position. Acta Chir Scand 1965;130:570-83.

2) Arnoldi CC. Venous pressure in patients with valvular incompetence of the veins of the lower limb. Acta Chir Scand 1966;132: $628-45$.

3) Recek C, Koudelka V. Effet circulatoire du reflux saphénien dans la varicose essentielle. [Impact of saphenous reflux on the venous circulation in varicose vein disease]. Phlébologie 1979;32:407-474.

4) Höjensgard IC, Stürup H. Static and dynamic pressures in superficial and deep veins of the lower extremity in man. Acta Physiol Scand 1952;21:49-61.

5) Recek C, Pojer H. Ambulatory pressure gradient in the veins of the lower extremity. VASA 2000; 29:187-90.
6) Lattimer CR, Azzam M, Kalodiki E, et al. Hemodynamic changes in the femoral vein with increasing outflow resistance. J Vasc Surg Venous Lymphat Disord 2014; 2:26-33.

7) Christopoulos D, Nicolaides AN, Cook A, et al. Pathogenesis of venous ulceration in relation to the calf muscle pump function. Surgery 1989;106:829-35.

8) Recek C: Saphena-Reflux als Ursache der venösen Zirkulationsstörung bei primärer Varikose mit chronischer Veneninsuffizienz. [Saphenous vein reflux is responsible for the venous circulatory disorder in primary varicose veins]. Acta Chir Austriaca 1998;2:76-77.

9) Recek C. Calf pump activity influencing venous hemodynamics in the lower extremity. Int J Angiol 2013;22:23-30.

10) Fitridge RA, Dunlop C, Raptis S, et al. A prospective randomized trial evaluating the haemodynamic role of incompetent calf perforating veins. Aust N Z J Surg 1999;69: 214-6. 
C Recek - Contribution of the calf pump and foot pump to the return of venous blood from the lower extremity

11) Padberg FT Jr, Pappas PJ, Araki CT, et al. Hemodynamic and clinical improvement after superficial vein ablation in primary combined venous insufficiency with ulceration. J Vasc Surg 1996;24:711-8.

12) Binns M, Pho RWH. Anatomy of the 'venous foot pump'. Injury 1988;19:443-5.
13) White JV, Katz ML, Cisek P, et al. Venous outflow of the leg: anatomy and physiologic mechanism of the plantar venous plexus. J Vasc Surg 1996;24:819-24.

14) Broderick BJ, Corley GJ, Quondamatteo F, et al. Venous emptying from the foot: influences of weight bearing, toe curls, electrical stimulation, passive compression, and posture. J Applied Physiol 2010;109:1045-52. 